\title{
Successful Treatment of Concurrent Mucormycosis and Diffuse Alveolar Hemorrhage in a Patient With Newly Diagnosed Systemic Lupus Erythematosus
}

\author{
Mi Ryoung $\mathrm{SEO}^{1}$, Jihong BAE${ }^{1}$, Jina $\mathrm{YEO}^{1}$, Hee Jung RYU ${ }^{1}$, Hyo-Jin $\mathrm{CHOI}^{1}$, Sangho $\mathrm{LEE}^{2}$, Han Joo BAEK \\ ${ }^{1}$ Department of Internal Medicine, Division of Rheumatology, Gachon University Gil Medical Center, Incheon, South Korea \\ ${ }^{2}$ Department of Pathology, Gachon University Gil Medical Center, Incheon, South Korea
}

Infection is often complicated with systemic lupus erythematosus (SLE) and is one of the leading causes of death in SLE patients. ${ }^{1}$ When SLE flare coexisting with serious infection is suspected, intensive immunosuppressive therapy is usually a challenge. Mucormycosis is a rare and rapidly progressing infection with high mortality. Diffuse alveolar hemorrhage (DAH) is also one of the rare and serious manifestations in SLE. ${ }^{2,3}$ We experienced an extremely rare case of SLE that was initially presented with $\mathrm{DAH}$ and mucormycosis concurrently.

A previously healthy, 42-year-old female patient was admitted with a one-month history of loss of appetite, weight loss, and rash on her face and extremities.

Complete blood count revealed leukopenia and mild anemia. Urinalysis showed microscopic hematuria and proteinuria. Both erythrocyte sedimentation rate and $\mathrm{C}$-reactive protein were slightly elevated. Immunologic studies were positive for antinuclear (homogeneous pattern, 1:1280), anti-Ro, anti-Sm, and antiribonucleoprotein antibodies, but anti-neutrophil cytoplasmic antibody was negative. Anti-double stranded deoxyribonucleic acid was elevated at $62.3 \mathrm{IU} / \mathrm{mL}$ and complements were low. Lupus anticoagulant and direct Coombs' test were positive. Abdominopelvic computed tomography (CT) showed multiple enlarged lymph nodes and borderline splenomegaly. Biopsies from skin lesions, right inguinal lymph nodes, and bone marrow were performed and their histopathology revealed leukocytoclastic vasculitis, reactive hyperplasia, and normal findings, respectively.

On the fifth day of hospitalization, she complained of cough and shortness of breath, and chest radiography showed consolidations in bilateral lower lung fields. Intravenous antibiotics were started for a presumed pulmonary infection. No microorganism was grown in the sputum, blood or urine, and further microbiologic tests were all negative for cytomegalovirus, aspergillus and Pneumocystis jirovecii. Highdose glucocorticoid was added for suspected concurrent lung involvement in newly developed SLE, but symptoms and lung infiltrations on CT (Figure 1a) worsened and black-colored crusted patches occurred on the right ala of her nose (Figure 2a). On the $17^{\text {th }}$ hospital day, the patient was transferred to the intensive care unit (ICU) and placed on mechanical ventilator due to expected impending respiratory failure. A bronchoscopic exam showed bloody bronchoalveolar lavage fluid. Biopsy of the nasal lesion and histopathology confirmed mucormycosis (Figure 2b). Plasma

\footnotetext{
Received: September 29, 2017 Accepted: November 02, 2017 Published online: January 15, 2018

Correspondence: Han Joo Baek, MD. Department of Internal Medicine, Division of Rheumatology, Gachon University Gil Medical Center, 21565 Incheon, South Korea. Tel: 82-32-460-3252 e-mail: baekhj@gilhospital.com

(02018 Turkish League Against Rheumatism. All rights reserved.
} 

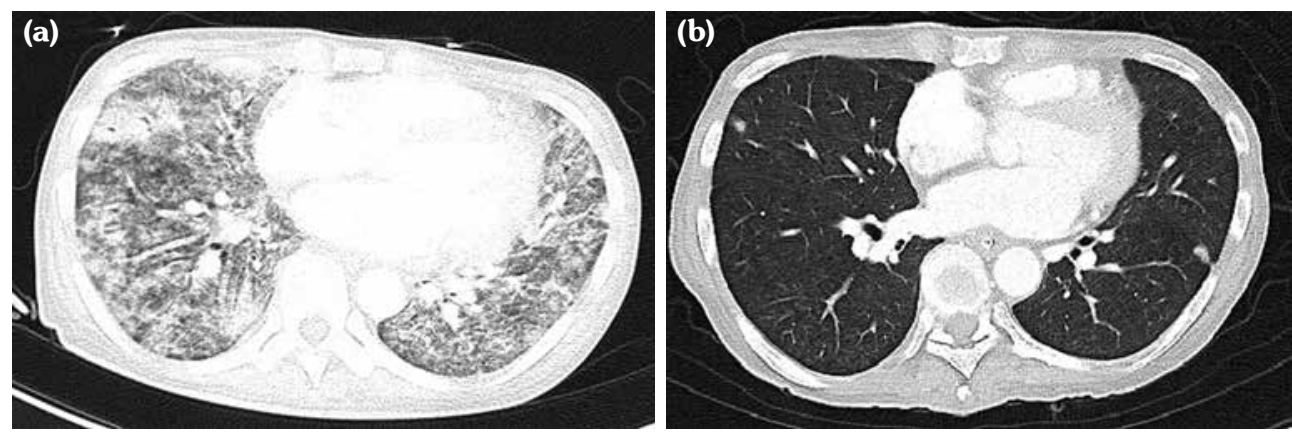

Figure 1. (a) Chest computed tomographs showed bilateral, diffuse, ill-defined, nodular consolidation and ground-glass opacification in lungs, suggesting diffuse alveolar hemorrhage. (b) The lung lesions were markedly resolved after cyclophosphamide and glucocorticoid pulse therapies.
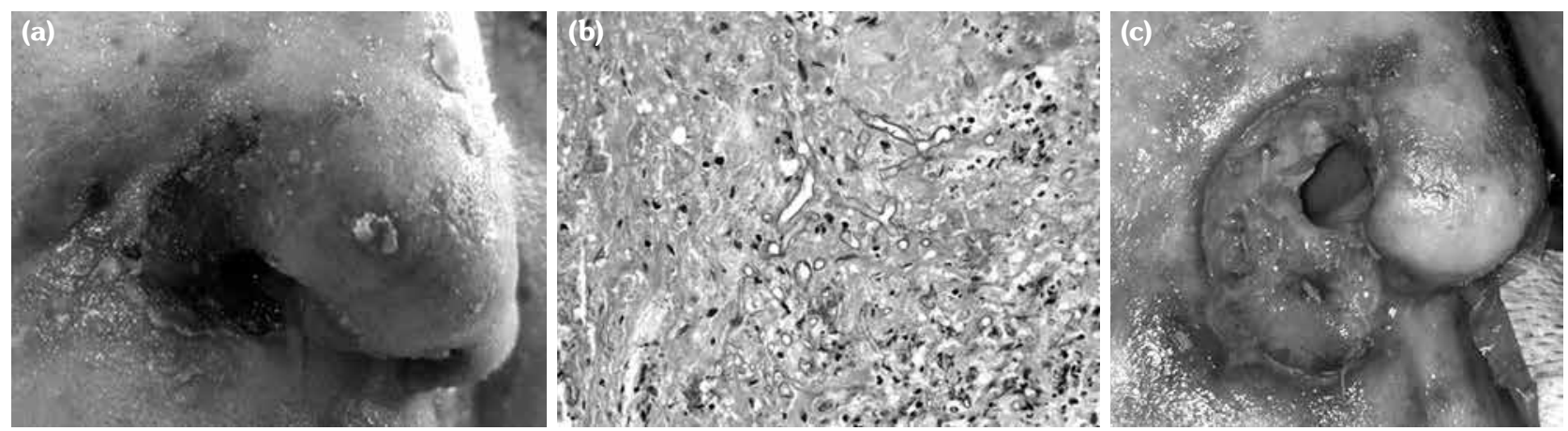

Figure 2. (a) Black-colored crusted patches on the right ala of nose were confirmed to be mucormycosis. (b) Periodic acid schiff staining of excised nose tissue showed thick fungal hyphae with right angle branching (magnification 400x) (c) Mucormycosis infected tissue was removed by surgical debridement.

exchange and intravenous immunoglobulin therapy were performed with liposomal amphotericin B. However, her respiration was not improved and the endotracheal tube was frequently blocked because of persistent DAH. Notwithstanding the risk of the concurrent infection aggravation, cyclophosphamide and glucocorticoid pulse therapy were added for the management of the refractory DAH. Three cycles of cyclophosphamide pulse therapies (intravenous cyclophosphamide $500 \mathrm{mg}$ with methylprednisolone $1 \mathrm{~g}$ ) were administered with a surgical debridement of the nasal mucormycosis lesions on the $39^{\text {th }}$ hospital day (Figure 2c). Her respiration stabilized and the lung lesion gradually resolved with the cyclophosphamide pulse therapies (Figure 1b). The patient eventually recovered after three months of ICU care.

Systemic lupus erythematosus patients with flare have an increased risk of infection. ${ }^{4,5}$ Thus, controlling SLE disease activity may be important for infection management. This case suggests that the prompt start of intensive immunosuppressive therapy with aggressive management of infection may result in a favorable outcome in SLE patients with a concurrence of flare and serious infection.

\section{Declaration of conflicting interests}

The authors declared no conflicts of interest with respect to the authorship and/or publication of this article.

\section{Funding}

The authors received no financial support for the research and/or authorship of this article.

\section{REFERENCES}

1. Fors Nieves CE, Izmirly PM. Mortality in Systemic Lupus Erythematosus: an Updated Review. Curr Rheumatol Rep 2016;18:21. 
2. Royer M, Puéchal X. Mucormycosis in systemic autoimmune diseases. Joint Bone Spine 2014;81:303-7.

3. Ednalino C, Yip J, Carsons SE. Systematic Review of Diffuse Alveolar Hemorrhage in Systemic Lupus Erythematosus: Focus on Outcome and Therapy. J Clin Rheumatol 2015;21:305-10.
4. Danza A, Ruiz-Irastorza G. Infection risk in systemic lupus erythematosus patients: susceptibility factors and preventive strategies. Lupus 2013;22:1286-94.

5. Bermas BL, Petri M, Goldman D, Mittleman B, Miller MW, Stocks NI, et al. T helper cell dysfunction in systemic lupus erythematosus (SLE): relation to disease activity. J Clin Immunol 1994;14:169-77. 